Artigo Original

\title{
Imagem corporal em universitários: associação com estado nutricional e sexo
}

\author{
Teresa Maria Bianchini de Quadros \\ Alex Pinheiro Gordia \\ Cilene Rebolho Martins \\ Diego Augusto Santos Silva \\ Elisa Pinheiro Ferrari \\ Édio Luiz Petroski
}

\begin{abstract}
Núcleo de Pesquisa em Cineantropometria e Desempenho Humano (NuCIDH) da Universidade Federal de Santa Catarina, Florianópolis, SC, Brasil
\end{abstract}

\begin{abstract}
Resumo: O presente estudo investigou a imagem corporal (IC) em universitários e sua relação com o estado nutricional e o sexo. Participaram do estudo 874 universitários (504 homens), com média de idade de $20,66(5,48)$ anos. A massa corporal e a estatura autorelatadas foram utilizadas para o cálculo do IMC. A IC foi avaliada através da escala de silhuetas corporais. O teste qui-quadrado foi utilizado para verificar associações, considerando p<0,05. A prevalência de insatisfação com a IC foi de $77,6 \%$, sendo que homens apresentaram maior insatisfação por magreza, enquanto que mulheres relataram maior insatisfação por excesso de peso $\left(X^{2}=89,68\right.$; $\left.p<0,001\right)$. Observou-se associação entre a IC e o estado nutricional tanto para homens $\left(X^{2}=146,20 ; p<0,001\right)$ quanto para mulheres $\left(X^{2}=91,88 ; p<0,001\right)$. $A$ prevalência de insatisfação com a IC dos universitários estudados foi elevada e apresentou-se associada ao sexo e ao estado nutricional.
\end{abstract}

Palavras-chave: Imagem corporal. Estado nutricional. Sexo. Estudantes.

Body image among university students: association with nutritional status and gender

\begin{abstract}
The present study investigated the body image (BI) of university students and its association with nutritional status and gender. A total of 874 students (504 men), with a mean age of 20.66 (5.48) years, participated in the study. Self-reported body weight and height were used for the calculation of BMI. Body image was evaluated using the body silhouette scale. Associations were analyzed using the chi-square test, with $\mathrm{p}<0.05$. The prevalence of $\mathrm{BI}$ dissatisfaction was $77.6 \%$, with men presenting greater dissatisfaction due to leanness, whereas women reported greater dissatisfaction because of excess weight $\left(X^{2}=89.68\right.$; $p<0.001)$. An association was observed between $B I$ and nutritional status in both men $\left(X^{2}=146.20 ; p<0.001\right)$ and women $\left(X^{2}=91.88 ; p<0.001\right)$. The prevalence of $\mathrm{BI}$ dissatisfaction was high among the university students studied and was associated with gender and nutritional status.
\end{abstract}

Key Words: Body image. Nutritional status. Gender. Students.

\section{Introdução}

A imagem corporal (IC) pode ser definida como uma ilustração que se tem na mente acerca do tamanho, da aparência e da forma do corpo, assim como das respostas emocionais a ele associados, sendo que a formação da IC está relacionada ao grau de precisão com que o tamanho do corpo é percebido e ao nível de satisfação ou rejeição corporal (SLADE, 1994). Com base neste conceito, evidencia-se que a IC é um importante componente da identidade pessoal.

Pesquisas recentes têm demonstrado que a insatisfação com a IC é observada tanto em indivíduos com transtornos alimentares (LAVOISY et al., 2008; GRILO et al., 2008) quanto em pessoas saudáveis de diferentes estratos da população (ALVES et al., 2008; ARROYO et al., 2008; GONCALVES et al., 2008; WRENCH; KNAPP, 2008). A prevalência deste fenômeno varia de $17,4 \%$ a $82,0 \%$ na população brasileira (BOSI et al., 2008; PINHEIRO; GIUGLIANI, 2006) e evidências de pesquisas internacionais indicam que esta prevalência tem aumentado com o passar dos anos (PIMENTA et al., 2009).

Os meios de comunicação de massa têm um papel fundamental no estabelecimento de um 
"padrão de beleza" da população (DERENNE; BERESIN, 2006). Atualmente, tem-se observado a introdução de um modelo de beleza intimamente associado à magreza entre as mulheres e corpos musculosos entre os homens. No entanto, este padrão de beleza é inalcançável para a maioria dos indivíduos, conduzindo a um distanciamento entre a IC ideal e a real, e quanto mais se distanciar da IC real, maior será a possibilidade de conflito, sentimentos negativos e incidência de baixa autoestima, afetando negativamente a saúde e a qualidade de vida (STEPHAN; FOUQUEREAU; FERNANDEZ, 2008; TABAK et al., 2007).

O Índice de Massa Corporal (IMC) é a medida mais comumente utilizada em estudos epidemiológicos para classificação do estado nutricional e indica se o peso de uma pessoa está dentro do recomendável para a saúde. Alguns estudos que visaram relacionar o estado nutricional, avaliado pelo IMC, com a IC observaram que indivíduos com excesso de peso foram mais suscetíveis para apresentar insatisfação com a IC (KAKESHITA; ALMEIDA, 2006; PINHEIRO; GIUGLIANI, 2006), enquanto que outras pesquisas evidenciaram que mesmo pessoas com massa corporal normal apresentaram alta incidência de insatisfação com o corpo (BOSI et al., 2008). Além disso, tem-se observado diferenças entre os sexos quanto à insatisfação com a IC. Há indícios de que as mulheres são mais insatisfeitas por excesso de peso, enquanto que os homens são mais insatisfeitos por magreza (DAMASCENO et al., 2005; KAKESHITA; ALMEIDA, 2006).

Estudos prévios relataram que a insatisfação com a IC é mais prevalente no final da adolescência e início da fase adulta (AL SABBAH et al., 2009; NISKAR et al., 2009). Dados do Instituto Nacional de Estudos e Pesquisas Educacionais Anísio Teixeira (INEP, 2006) evidenciam que a maioria dos universitários brasileiros ingressa no ensino superior com idades entre 18 a 24 anos. Neste sentido, estudantes ingressantes em universidades parecem constituir um grupo de risco para este desfecho, considerando-se que a prevalência de insatisfação com a IC entre universitários brasileiros se aproxima de 80\% (COQUEIRO et al., 2008; GONCCALVES et al., 2008). Entretanto, não se tem conhecimento de nenhum estudo com amostra representativa de estudantes de uma universidade brasileira, o que impede maiores inferências sobre o assunto.

Diante das lacunas presentes na literatura, o objetivo do presente estudo foi investigar a prevalência de insatisfação com a IC em uma amostra representativa de estudantes ingressantes em uma universidade pública brasileira, bem como sua relação com o estado nutricional e o sexo.

\section{Métodos \\ População e Amostra}

Este estudo foi realizado com base no banco de dados do projeto de pesquisa "Avaliação da aptidão física relacionada à saúde de universitários da Universidade Federal de Santa Catarina (UFSC)". O protocolo do estudo foi aprovado pelo Comitê de Ética em Pesquisa da Universidade Federal de Santa Catarina (processo $n^{\circ}$ 096/2007).

A população de estudo foi composta por 2290 universitários ingressantes em uma universidade pública da região Sul do Brasil, no primeiro semestre do ano letivo de 2008. Para o cálculo do tamanho da amostra representativa da população foi utilizada a metodologia recomendada por Thomas; Nelson e Silverman (2007). Considerouse um nível de confiança igual a 95\% e um erro máximo permitido de 3,0 pontos percentuais. Utilizou-se a amostragem estratificada proporcional por centro de ensino e turno de estudo. Para o sorteio das turmas dentro de cada centro de ensino, utilizou-se o procedimento randomizado. Com base no cálculo amostral, estimou-se que seria necessário avaliar 728 universitários, no entanto, devido à coleta de dados por conglomerado participaram do estudo 921 indivíduos. Foram excluídos os indivíduos com dados incompletos $(n=47)$. Desta forma, a amostra final do presente estudo foi composta por 874 universitários (504 do sexo masculino e 370 do sexo feminino).

\section{Instrumentos e Procedimentos}

A massa corporal e a estatura autorelatadas foram utilizadas para o cálculo do IMC. Para a classificação do IMC, utilizaram-se os pontos de corte estabelecidos pela World Health Organization (WHO, 1995). Devido ao poder da análise estatística, as classificações de sobrepeso e obesidade foram agrupadas, caracterizando o excesso de peso. 
As informações referentes à percepção da IC foram obtidas por autoavaliação, utilizando-se a escala de nove silhuetas corporais propostas por Stunkard; Sorenson e Schlusinger (1983). O conjunto de silhuetas foi mostrado aos universitários, seguido das perguntas: 1) Qual a silhueta que melhor representa sua aparência física atual (real?); 2) Qual silhueta você gostaria de ter (ideal?). Para verificar a insatisfação com a IC utilizou-se a diferença entre a silhueta real e a ideal. Quando a diferença foi igual à zero, o indivíduo foi classificado como satisfeito, e se diferente de zero, como insatisfeito. Quando a diferença foi positiva, o indivíduo foi considerado como insatisfeito por excesso de peso, e quando negativa, como insatisfeito por magreza.

\section{Análise Estatística}

Recorreu-se a análise descritiva das variáveis através de indicadores estatísticos de tendência central (média), variabilidade (desvio padrão) e frequências percentuais. Visando analisar a associação da IC com o estado nutricional e com o sexo, foi empregado o teste do qui-quadrado. $O$ nível de significância foi estabelecido em $p<0,05$.

\section{Resultados}

As características descritivas da amostra investigada de acordo com o sexo podem ser observadas na tabela 1.

Tabela 1. Características descritivas da amostra estudada com valores expressos em média e desvio padrão de acordo com o sexo.

\begin{tabular}{lcc}
\hline \multicolumn{1}{c}{ Variáveis } & $\begin{array}{c}\text { Masculino } \\
\text { Média (DP) }\end{array}$ & $\begin{array}{c}\text { Feminino } \\
\text { Média (DP) }\end{array}$ \\
\hline Idade (anos) & $20,4(5,0)$ & $20,9(6,0)$ \\
Peso corporal $(\mathrm{kg})$ & $71,7(11,8)$ & $57,3(9,0)$ \\
Estatura $(\mathrm{cm})$ & $177,4(7,3)$ & $164,1(6,3)$ \\
IMC $\left(\mathrm{kg} \cdot \mathrm{m}^{-2}\right)$ & $22,7(3,1)$ & $21,3(3,0)$ \\
\hline
\end{tabular}

$\mathrm{Na}$ tabela 2, pode-se verificar a associação entre o estado nutricional e o sexo na amostra estudada. Os achados indicaram que a maioria dos universitários encontrava-se com peso normal. Entretanto, uma parcela significante da amostra apresentou baixo peso (8,0\%) e excesso de peso $(15,4 \%)$. No que se refere ao sexo, o excesso de peso foi mais prevalente entre homens e o baixo peso entre as mulheres $\left(X^{2}=36,59 ; p<0,001\right)$.
Tabela 2. Associação entre o estado nutricional e o sexo em universitários.

\begin{tabular}{|c|c|c|c|}
\hline \multirow[b]{2}{*}{$\begin{array}{c}\text { Estado } \\
\text { Nutricional }\end{array}$} & \multicolumn{2}{|c|}{ Sexo } & \multirow[b]{2}{*}{$\begin{array}{l}\text { Total } \\
\%(n)\end{array}$} \\
\hline & $\begin{array}{c}\text { Masculino } \\
\%(n)\end{array}$ & $\begin{array}{c}\text { Feminino } \\
\%(n)\end{array}$ & \\
\hline Baixo peso & $4,0(20)$ & $13,5(50)$ & $8,0(70)$ \\
\hline Normal & $76,6(386)$ & 76,5 (283) & 76,5 (669) \\
\hline Excesso de peso & $19,4(98)$ & $10,0(37)$ & $15,4(135)$ \\
\hline
\end{tabular}

A prevalência de insatisfação com a IC entre os universitários investigados foi de $77,6 \%$, sendo maior a insatisfação por excesso de peso do que por magreza (46,1\% vs. 31,5\%). Quanto ao sexo, pode-se observar (tabela 3 ) que indivíduos do sexo masculino apresentaram maior prevalência de insatisfação por magreza, enquanto que seus pares do sexo feminino relataram maior insatisfação por excesso de peso $\left(X^{2}=89,68\right.$; $p<0,001)$.

Tabela 3. Associação entre a IC e o sexo em universitários.

\begin{tabular}{|c|c|c|c|}
\hline \multirow{2}{*}{$\begin{array}{l}\text { Imagem } \\
\text { Corporal }\end{array}$} & \multicolumn{2}{|c|}{ Sexo } & \multirow{2}{*}{$\begin{array}{l}\text { Total } \\
\%(n)\end{array}$} \\
\hline & $\begin{array}{c}\text { Masculino } \\
\%(n)\end{array}$ & $\begin{array}{c}\text { Feminino } \\
\%(n)\end{array}$ & \\
\hline $\begin{array}{l}\text { Insatisfeito } \\
\text { por magreza }\end{array}$ & 43,3 (218) & $15,4(57)$ & 31,5 (275) \\
\hline Satisfeito & 22,6 (114) & $22,2(82)$ & $22,4(196)$ \\
\hline $\begin{array}{l}\text { Insatisfeito } \\
\text { por excesso }\end{array}$ & 34,1 (172) & $62,4(231)$ & $46,1(403)$ \\
\hline
\end{tabular}

Nota: $\mathrm{X}^{2}=89,68 ; \mathrm{p}<0,001$

Os resultados da tabela 4 indicaram associação entre a IC e o estado nutricional tanto para o sexo masculino $\left(X^{2}=146,20 ; \quad p<0,001\right)$ quanto para o feminino $\left(X^{2}=91,88 ; p<0,001\right)$. No entanto, nota-se que uma grande proporção de universitários do sexo masculino que relatou insatisfação por magreza apresentou massa corporal normal, enquanto que a maioria das universitárias do sexo feminino que relatou insatisfação por excesso de peso foi considerada com peso normal de acordo com a classificação do IMC. 
Tabela 4. Associação entre a IC e o estado nutricional em universitários de acordo com o sexo.

\begin{tabular}{|c|c|c|c|c|}
\hline \multirow[b]{2}{*}{ Imagem Corporal } & \multicolumn{3}{|c|}{ Estado Nutricional } & \multirow{2}{*}{$\begin{array}{c}\text { Estatística } X^{2} \\
p \text { valor }\end{array}$} \\
\hline & $\begin{array}{c}\text { Baixo peso } \\
\%(\mathrm{n})\end{array}$ & $\begin{array}{l}\text { Normal } \\
\%(n)\end{array}$ & $\begin{array}{c}\text { Excesso de peso } \\
\%(n)\end{array}$ & \\
\hline \multicolumn{5}{|c|}{ Masculino } \\
\hline Insatisfeito por magreza & $8,3(18)$ & $90,8(198)$ & $0,9(2)$ & \multirow{3}{*}{$\begin{array}{r}X^{2}=146,20 \\
p<0,001\end{array}$} \\
\hline Satisfeito & $1,8(2)$ & $85,1(97)$ & $13,2(15)$ & \\
\hline Insatisfeito por excesso & $0(0)$ & $52,9(91)$ & $47,1(81)$ & \\
\hline \multicolumn{5}{|c|}{ Feminino } \\
\hline Insatisfeito por magreza & $45,6(26)$ & $54,4(31)$ & $0(0)$ & \multirow{3}{*}{$\begin{array}{l}x^{2}=91,88 \\
p<0,001\end{array}$} \\
\hline Satisfeito & $22,0(18)$ & $76,8(63)$ & $1,2(1)$ & \\
\hline Insatisfeito por excesso & $2,6(6)$ & $81,8(189)$ & $15,6(36)$ & \\
\hline
\end{tabular}

\section{Discussão}

Considerando estudos prévios, não se tem conhecimento de outra pesquisa que tenha investigado a insatisfação com a IC e sua associação com o estado nutricional e sexo em uma amostra representativa de acadêmicos de uma universidade brasileira. Desta forma, os dados do presente estudo podem contribuir para o avanço do conhecimento sobre o tema, podendo ser utilizados em programas de promoção à saúde e qualidade de vida nos campi.

Os achados do presente estudo indicaram que $77,6 \%$ dos universitários estudados relataram insatisfação com a IC. Estes dados corroboram prevalências descritas em pesquisas com universitários (COQUEIRO et al., 2008; GONÇALVES et al., 2008) e foram um pouco superiores aos relatados para a população brasileira em geral (TRICHES; GIUGLIANI, 2007; DAMASCENO et al., 2005). Este panorama é preocupante e conduz a reflexões acerca de estratégias que visem aumentar a aceitação corporal da população, tendo em vista a consistente associação entre insatisfação com a IC e depressão, baixa autoestima e percepção negativa da qualidade de vida (STEPHAN; FOUQUEREAU; FERNANDEZ, 2008; TABAK et al., 2007).

O excesso de peso tem sido apontado como um problema de saúde pública. Estimativas da WHO (2005) indicam que 2,6 milhões de pessoas morrem todo o ano como resultado do excesso de peso. No Brasil, há evidências de que o custo para a saúde pública com internações decorrentes da obesidade atingiu, aproximadamente, 20 milhões de dólares em 2001 (SICHIERI; NASCIMENTO; COUTINHO, 2007), e estes valores, possivelmente, são superiores atualmente. A prevalência de excesso de peso dos universitários estudados foi de 15,4\%. Estes achados foram inferiores aos observados em levantamentos epidemiológicos nacionais e internacionais (IBGE, 2004; MINISTÉRIO DA SAÚDE, 2009; FLEGAL et al., 2002), porém, superiores aos relatados em pesquisas realizadas com universitários (VARGAS-ZÁRATE; BECERRA-BULLA; PRIETO-SUÁREZ, 2008; MARCONDELLI; COSTA; SCHMITZ, 2008), evidenciando a necessidade de projetos institucionais de intervenção e prevenção para o controle da massa corporal na amostra estudada.

Os achados do presente estudo indicaram associação entre a IC e o estado nutricional, corroborando a literatura (KAKESHITA; ALMEIDA, 2006; PINHEIRO; GIUGLIANI, 2006; BOSI et al., 2008). No entanto, nota-se que uma grande proporção dos universitários investigados, classificada como peso normal de acordo com o IMC, relatou insatisfação com a IC, indicando contradições entre a avaliação de saúde e da estética. Essas divergências, possivelmente, estão relacionadas a uma preocupação exagerada com a estética corporal imposta pela mídia, fazendo com que o padrão de beleza idealizado não corresponda aos padrões considerados adequados para a saúde. 
No que refere a associação entre a IC e o sexo, observou-se que a prevalência de insatisfação por excesso de peso foi mais evidente em mulheres enquanto que uma maior proporção de homens relatou insatisfação por magreza, independente do estado nutricional. Estes achados são semelhantes aos observados em outros estudos (DAMASCENO et al., 2005; KAKESHITA; ALMEIDA, 2006). No que se refere à saúde da população, este desejo das mulheres pela redução do tamanho da silhueta corporal pode contribuir para a ocorrência de distúrbios alimentares (como a anorexia nervosa e a bulimia) (STRIEGEL-MOORE et al., 2009), enquanto que para homens observa-se preferência por um corpo mais volumoso, fato que pode estimular um transtorno comportamental denominado vigorexia (caracterizado pela prática excessiva de exercícios físicos, obsessiva preocupação com o corpo e adoção de práticas alimentares não convencionais) (MOSLEY, 2009). Este panorama conduz a reflexões acerca de estratégias para aumentar a aceitação do próprio corpo na população universitária por meio de projetos que visem à construção do conhecimento sobre temas relacionados à saúde e bem estar.

O presente estudo possui limitações que merecem detalhamento: 1) trata-se de uma pesquisa com delineamento transversal e por este motivo, possui baixa capacidade para estabelecer relações de causa e efeito, tendo em vista que a coleta de dados sobre a exposição e o desfecho foi realizada simultaneamente e, portanto, dificulta o conhecimento da relação temporal existente entre eles. No entanto, acredita-se que este delineamento foi satisfatório para abordar o problema de pesquisa do presente estudo, pois de acordo com Bastos e Duquia (2007), estudos com este desenho são recomendados quando se deseja estimar a frequência com que um determinado evento de saúde se manifesta em uma população específica, além dos fatores associados com o mesmo; 2) o instrumento utilizado para coletar as informações referentes à IC apresenta desenhos das silhuetas em formas lineares bidimensionais, podendo implicar falhas na representação total do corpo e na distribuição da gordura, afetando na formação individual da IC. Entretanto, a utilização deste instrumento se justifica devido à facilidade e praticidade para aplicação, pela boa fidedignidade e frequente utilização em estudos epidemiológicos (SÁNCHEZ-VILLEGAS et al., 2001; PINHEIRO; GIUGLIANI, 2006) e com a população universitária (KAKESHITA; ALMEIDA, 2006; COQUEIRO et al., 2008); e 3) a avaliação da massa corporal e da estatura por meio do autorelato pode ter subestimado ou superestimado o IMC dos universitários, havendo a possibilidade de interferência nos achados. Contudo, estudos recentes indicam a validade deste método em estudos populacionais para fornecer informações confiáveis do estado nutricional da população adulta brasileira (COQUEIRO et al., 2009; SILVEIRA et al., 2005). Além disso, este procedimento tem sido extensivamente utilizado para avaliação do estado nutricional de universitários (BRUNT; RHEE, 2008; HOLM-DENOMA et al., 2008).

Em conclusão, a prevalência de insatisfação com a IC dos universitários estudados foi elevada e apresentou-se associada ao sexo e ao estado nutricional. Estudos com delineamento longitudinal são encorajados para confirmar os achados do presente estudo e contribuir para o avanço do conhecimento sobre a percepção da IC e fatores associados em universitários. Além disso, outras variáveis comportamentais relacionadas aos padrões de estética e a IC podem ser consideradas em futuras investigações, tais como: influência da mídia, amigos e familiares, depressão, autoestima, comportamento alimentar, uso de esteróides anabolizantes e dependência de exercício.

\section{Referências}

AL SABBAH, H.; VERERCKEN, C. A.; ELGAR, F. J.; NANSEL, T.; AASVEE, K.; ABDEEN, Z.; OJALA, K.; AHLUWALIA, N.; MAES, L. Body weight dissatisfaction and communication with parents among adolescents in 24 countries: international cross-sectional survey. BMC Public Health, v. 52, n. 9, 2009. http://dx.doi.org/10.1186/1471-2458-9-52

ALVES, E.; VASCONCELOS, F. A. G.; CALVO, M. C. M.; NEVES, J. Prevalência de sintomas de anorexia nervosa e insatisfação com a imagem corporal em adolescentes do sexo feminino do 
Município de Florianópolis, Santa Catarina, Brasil. Cadernos de Saúde Pública, Rio de Janeiro, v. 24, n. 3, p. 503-512, 2008.

http://dx.doi.org/10.1590/S0102311X2008000300004

ARROYO, M.; GONZALEZ-DE-SUSO, J. M.; SANCHEZ, C.; ANSOTEGUI, L.; ROCANDIO, A. M. Body image and body composition: comparisons of young male elite soccer players and controls. International Journal of Sport Nutrition and Exercise Metabolism, v. 18, n. 6, p. 628-638, 2008.

BASTOS, J. L. D.; DUQUIA, R. P. Um dos delineamentos mais empregados em epidemiologia: estudo transversal. Scientia Medica, Porto Alegre, v. 17, n. 4, p. 229-232, 2007. Disponível em:

http://revistaseletronicas.pucrs.br/ojs/index.php/sc ientiamedica/article/viewFile/2806/2634.

BOSI, M. L. M.; LUIZ, R. R.; UCHIMURA, K. Y.; OLIVEIRA, F. P. Comportamento alimentar e imagem corporal entre estudantes de educação física. Jornal Brasileiro de Psiquiatria, Rio de Janeiro, n. 57, n. 1, p. 28-33, 2008.

http://dx.doi.org/10.1590/S004720852008000100006

BRUNT, A. R.; RHEE, Y. S. Obesity and lifestyle in U.S. college students related to living arrangemeents. Appetite, n. 51, v. 615-621, 2008.

http://dx.doi.org/10.1016/j.appet.2008.04.019

COQUEIRO, R. S.; PETROSKI, E. L.; PELEGRINI, A.; BARBOSA, A. R. Insatisfação com a imagem corporal: avaliação comparativa da associação com estado nutricional em universitários. Revista de Psiquiatria do Rio Grande do Sul, Porto Alegre, n. 30, v. 1, p. 3138, 2008. Disponível em: http://www.scielo.br/pdf/rprs/v30n1/v30n1a09.pdf.

COQUEIRO, R. S.; BORGES, L. J.; ARAÚJO, V. C.; PELEGRINI, A.; BARBOSA, A. R. Medidas auto-referidas são válidas para avaliação do estado nutricional na população brasileira? Revista Brasileira de Cineantropometria e Desempenho Humano, Florianópolis, v. 11, n. 1, p. 113-119, 2009. Disponível em: http://www.rbcdh.ufsc.br/DownloadArtigo.do?artig $\underline{0=480}$.

DAMASCENO, V. O.; LIMA, J. R. P.; VIANNA, J. M.; VIANNA, V. R. A.; NOVAES, J. S. Tipo físico ideal e satisfação com a imagem corporal de praticantes de caminhada. Revista Brasileira de Medicina do Esporte, Niterói, v. 11, n. 3, p. 181186, 2005. http://dx.doi.org/10.1590/S151786922005000300006
DERENNE, J. L.; BERESIN, E. V. Body image, media, and eating disorders. Academic Psychiatry, v. 30, n. 3, p. 257-261, 2006. http://dx.doi.org/10.1176/appi.ap.30.3.257

FLEGAL, K. M.; CARROLL, M. D.; OGDEN, C. L.; JOHNSON, C. L. Prevalence and trends in obesity among US adults, 1999-2000. JAMA, v. 288, n. 14, p. 1723-1727, 2002. Disponível em: http://jama.ama-assn.org/cgi/reprint/288/14/1723.

GONÇALVES, T. D.; BARBOSA, M. P.; ROSA, L. C. L.; RODRIGUES, A. M. Comportamento anoréxico e percepção corporal em universitários. Jornal Brasileiro de Psiquiatria, Rio de Janeiro, v. 57, n. 3, p. 166-170, 2008.

http://dx.doi.org/10.1590/S004720852009000300002.

GRILO, C. M.; HRABOSKY, J. I.; WHITE, M. A.; ALLISON, K. C.; STUNKARD, A. J.; M.; ROBIN, $M$. Overvaluation of shape and weight in binge eating disorder and overweight controls: Refinement of a diagnostic construct. Journal of Abnormal Psychology, v. 117, n. 2, p. 414-419, 2008.

HOLM-DENOMA, J. M.; JOINER, T. E.; VOHS, K. D.; HEATHERTON, T. F. The "freshman fifteen" (the "freshman five" actually): predictors and possible explanations. Health Psychology, v. 27 (Supl. 1), p. S3-S9, 2008.

\section{INSTITUTO BRASILEIRO DE GEOGRAFIA E ESTATÍSTICA - IBGE. Pesquisa de} Orçamentos Familiares (POF) 2002-2003. Disponível em: http://www.ibge.gov.br. Acesso em: 23 jun. 2007.

KAKESHITA, I. S.; ALMEIDA, S. S. Relação entre índice de massa corporal e a percepção da autoimagem em universitários. Revista de Saúde Pública, São Paulo, v. 40, n. 3, p. 497-504, 2006. http://dx.doi.org/10.1590/S0034$\underline{89102006000300019}$

LAVOISY, G.; GUELFI, J. D.; VERA, L.; DARDENNES, R.; ROUILLON, F. Evaluation of perturbed body image in eating disorders using the Body Shape Questionnaire. Encephale, v. 34, n. 6, p. 570-576, 2008.

http://dx.doi.org/10.1016/i.encep.2007.11.005

MARCONDELLI, P.; COSTA, T. H. M.; SCHMITZ, B. A. S. Nível de atividade física e hábitos alimentares de universitários do $3^{\circ}$ ao $5^{\circ}$ semestres da área da saúde. Revista de Nutrição, Campinas, v. 21, n. 1, p. 39-47, 2008. http://dx.doi.org/10.1590/S1415$\underline{52732008000100005 .}$.

MINISTÉRIO DA EDUCAÇÃO. INSTITUTO NACIONAL DE ESTUDOS E PESQUISAS 
EDUCACIONAIS ANÍSIO TEIXEIRA. Sinopses

Estatísticas da Educação Superior-

Graduação, 2006. Disponível em:

http://www.inep.gov.br/superior/censosuperior/sin

opse. Acesso em: 05 abr. 2009.

MINISTÉRIO DA SAÚDE. Vigilância de fatores

de risco e proteção para doenças crônicas por inquérito telefônico (VIGITEL). Brasília:

Ministério da Saúde, 2009. Disponível em: http://bvsms.saude.gov.br/bvs/publicacoes/vigitel 2008.pdf. Acesso em: 15 abr. 2009.

MOSLEY, P. E. Bigorexia: bodybuilding and muscle dysmorphia. European Eating Disorders Review, v. 17, n. 3, p. 191-198, 2009.

http://dx.doi.org/10.1002/erv.897.

NISKAR, A.; BARON-EPEL, O.; GARTYSANDALON, N.; KEINAN-BOKER, L. Body weight dissatisfaction among Israeli Jewish and Arab women with normal or overweight-obese body mass index, Israeli INHIS-1, 2003-2004.

Preventing Chronic Disease, v. 6, n. 2, p. A51, 2009. Disponível em:

http://www.cdc.gov/pcd/issues/2009/Apr/pdf/08 0 118.pdf.

PIMENTA, A. M.; SANCHEZ-VILLEGAS, A.; BESRASTROLLO, M.; LOPEZ, C. N.; MARTINEZGONZALEZ, M. A. Relationship between body image disturbance and incidence of depression: the SUN prospective cohort. BMC Public Health, v. 9, n. 1, 2009. http://dx.doi.org/10.1186/14712458-9-1.

PINHEIRO, A. P.; GIUGLIANI, E. R. J. Insatisfação corporal em escolares no Brasil: prevalência e fatores associados. Revista de Saúde Pública, São Paulo, v. 40, n. 3, p. 489496, 2006. http://dx.doi.org/10.1590/S003489102006000300018

SÁNCHEZ-VILLEGAS, A.; MADRIGAL, H.; MARTÍNEZ-GONZÁLEZ, M. A.; KEARNEY, J.; GIBNEY, M. J.; IRALA, J.; MARTÍNEZ, J. A. Perception of body image as indicator of weight status in the European Union. Journal of Human Nutrition and Dietetics, v. 14, n. 2, p. 93-102, 2001. http://dx.doi.org/10.1046/i.1365277X.2001.00281.X.

SICHIERI, R.; NASCIMENTO, S.; COUTINHO, W. The burden of hospitalization due to overweight and obesity in Brazil. Cadernos de Saúde Pública, Rio de Janeiro, v. 23, n. 7, p. 1721-1727, 2007. http://dx.doi.org/10.1590/S0102$\underline{311 \times 2007000700025}$

SILVEIRA, E. A.; ARAÚJO, C. L.; GIGANTE, D. P.; BARROS, A. J. D.; LIMA, M. S. Validação do peso e altura referidos para o diagnóstico do estado nutricional em uma população de adultos no Sul do Brasil. Cadernos de Saúde Pública, Rio de Janeiro, v. 21, n. 1, p. 235-245, 2005. http://dx.doi.org/10.1590/S0102311X2005000100026.

SLADE, P. D. What is body image? Behaviour Research and Therapy, v. 32, n. 5, p. 497-502, 1994. http://dx.doi.org/10.1016/00057967(94)90136-8.

STEPHAN, Y.; FOUQUEREAU, E.; FERNANDEZ, A. Body satisfaction and retirement satisfaction: the mediational role of subjective health. Aging and Mental Health, v. 12, n. 3, p. 374-381, 2008. http://dx.doi.org/10.1080/13607860802120839.

STRIEGEL-MOORE, R. H.; ROSSELLI, F.; PERRIN, N.; DEBAR, L.; WILSON, G. T.; MAY, A.; KRAEMER H. C. Gender difference in the prevalence of eating disorder symptoms.

International Journal of Eating Disorders. No prelo. DOI: 10.1002/eat.20625.

STUNKARD, A.J.; SORENSEN, T.;

SCHULSINGER, F. Use of the Danish Adoption Register for the study of obesity and thinness. In: KETY, S.S.; ROWLAND, L.P.; SIDMAN, R.L.; MATTHYSSE, S.W. (Ed.). The Genetics of Neurological and Psychiatric Disorders. New York: Raven Press, 1983. p. 115-120.

TABAK, I.; MAZUR, J.; OBLACINSKA, A.; JODKOWSKA, M. Body mass, self-esteem and life satisfaction in adolescents aged 13-15 years. Medycyna Wieku Rozwojowego, n. 11, v. $3 \mathrm{Pt}$ 1, p. 281-90, 2007.

THOMAS, J. R.; NELSON, J. K.; SILVERMAN, S. J. Métodos de Pesquisa em Atividade Física. 5 ed. Porto Alegre: Artmed, 2007.

TRICHES, R. M.; GIUGLIANI, E. R. J. Insatisfação corporal em escolares de dois municípios da região Sul do Brasil. Revista de Nutrição, Campinas, n. 20, v. 2, p. 119-128, 2007. http://dx.doi.org/10.1590/S141552732007000200001.

VARGAS-ZÁRATE, M.; BECERRA-BULLA, F.; PRIETO-SUÁREZ, E. Evaluación antropométrica de estudiantes universitarios em Bogotá,

Colômbia. Revista de Salud Pública, n. 10, v. 3, p. 433-442, 2008. Disponível em:

http://www.scielo.org.co/pdf/rsap/v10n3/v10n3a08 .pdf.

WORLD HEALTH ORGANIZATION. WHO Expert Committee on Physical Status: The use and interpretation of anthropometry physical status. Geneva: World Health Organization, 1995 (WHO Technical Report Series, v. 854).

Disponível em: 
http://whqlibdoc.who.int/trs/WHO TRS 854.pdf.

Acesso em: 10 out. 2004.

WORLD HEALTH ORGANIZATION. Preventing

chronic disease: a vital investment. Geneva:

World Health Organization, 2005. Disponível em:

http://www.who.int/chp/chronic disease report/full report.pdf. Acesso em: 15 jun. 2006.

WRENCH, J. S.; KNAPP, J. L. The effects of body image perceptions and sociocommunicative orientations on self-esteem, depression, and identification and involvement in the gay community. Journal of Homosexuality, n. 55, v. 3, p. 471-503, 2008

\section{Endereço:}

Teresa Maria Bianchini de Quadros

Rua Barão do Rio Branco, 1124 - Centro

Lapa PR Brasil

83.750-000

Telefone: (41) 3622-5862

e-mail: tetemb@gmail.com

Recebido em: 31 de maio de 2009.

Aceito em: 9 de novembro de 2009.

\section{(c)}

Motriz. Revista de Educação Física. UNESP, Rio Claro, SP, Brasil - elSSN: 1980-6574 - está licenciada sob Licenca Creative Commons 